\title{
Do Words Stink? Neural Reuse as a Principle for Understanding Emotions in Reading
}

\author{
Johannes C. Ziegler ${ }^{1}$, Marie Montant ${ }^{1}$, Benny B. Briesemeister ${ }^{2}$, Tila T. Brink ${ }^{2}$, \\ Bruno Wicker ${ }^{1}$, Aurélie Ponz ${ }^{1}$, Mireille Bonnard ${ }^{3}$, \\ Arthur M. Jacobs ${ }^{2}$, and Mario Braun ${ }^{4}$
}

\begin{abstract}
How do we understand the emotional content of written words? Here, we investigate the hypothesis that written words that carry emotions are processed through phylogenetically ancient neural circuits that are involved in the processing of the very same emotions in nonlanguage contexts. This hypothesis was tested with respect to disgust. In an fMRI experiment, it was found that the same region of the left anterior insula responded whether people observed facial expressions of disgust or whether they read
\end{abstract}

\section{INTRODUCTION}

How do people understand the emotional content of a written word? Classic psycholinguistic theories would argue that emotional content is not processed any different from other forms of meaning. According to this view, the extraction of emotional content would happen in the classic reading network that includes occipito-temporal areas in charge of visual and orthographic analyses (Dehaene \& Cohen, 2011) that project onto spoken language areas in temporal and frontal cortex (e.g., Wernicke, Broca) in charge of processing meaning and phonology (Price, 2012). Thus, computationally speaking, understanding what it means for somebody to be "happy" would not be any different from understanding what it means to be "alive" or "tall." In both cases, meaning is processed through the computation or analysis of abstract semantic properties or features (Mahon \& Caramazza, 2008; Pexman, Holyk, \& Monfils, 2003; McRae, de Sa, \& Seidenberg, 1997). This could be called the "cold" view of reading (Jacobs et al., 2015; Jacobs \& Ziegler, 2015).

In line with more neurobiological theories of language mechanisms (Pulvermuller, 1999, 2005), one could argue that understanding emotional content during reading might rely on the activation of neural structures normally involved in our own personally experienced emotion. For

\footnotetext{
${ }^{1}$ Aix-Marseille University and CNRS, France, ${ }^{2}$ Freie Universität Berlin, ${ }^{3}$ Aix-Marseille University and INSERM, France, ${ }^{4}$ University of Salzburg, and Centre for Cognitive Neuroscience
}

words with disgusting content. In a follow-up experiment, it was found that repetitive TMS over the left insula in comparison with a control site interfered with the processing of disgust words to a greater extent than with the processing of neutral words. Together, the results support the hypothesis that the affective processes we experience when reading rely on the reuse of phylogenetically ancient brain structures that process basic emotions in other domains and species. example, understanding what it means to be "angry" would depend on our ability to activate neural structures involved in our personal experience of anger, such as the amygdala (LaBar, Gatenby, Gore, LeDoux, \& Phelps, 1998). This idea is most clearly articulated in the context of theories of embodied cognition (Barsalou, 2008; Niedenthal, 2007), mirror neurons (Gallese, Keysers, \& Rizzolatti, 2004), or neural reuse (Anderson, 2010). The core idea is that the semantic neural network that allows us to compute the meaning of words should include the one that allows us to compute meaning in semantically similar nonlinguistic contexts. Hypothetically, word recognition is made possible through simulations that are consisted in the reenactment of stored perceptual, motor, and introspective information acquired during development in a variety of body-environment interactions. This could be viewed as the "hot" view of reading (Jacobs \& Ziegler, 2015).

With respect to the neural correlates of reading emotion words, three studies seem particularly relevant in the context of this study. First, in an fMRI experiment, González et al. (2006) asked participants to passively read odor-related words, such as "garlic," "cinnamon," or "jasmine." In line with the embodied cognition perspective, they found that odor-related words, compared with neutral words, activated the primary olfactory cortex, which includes the piriform cortex and the amygdala. Second, in a somewhat similar study, Barrós-Loscertales et al. (2012) asked participants to read words with gustatory links primarily related to taste (e.g., "salt"). They found 
that taste-related words strongly activated primary and secondary gustatory cortices, including the anterior insula, frontal operculum, and lateral orbitofrontal gyrus. These studies were taken to suggest that the meaning of taste or odor words is grounded in distributed cortical circuits that include the primary areas that process these sensations. Finally, Ponz et al. (2014) presented participants with disgust-related and neutral words while recording ERPs. They found differences between disgust and neutral words as early as $200 \mathrm{msec}$, which were localized in the left anterior insula, a region that is specifically activated in relation to disgust (see below). Similar differences were found in the intracranial recordings of two epileptic patients with depth electrodes in IC and OFC.

The goal of this study was to test the embodied view of word perception by investigating whether people would activate the same primary emotion network when they process emotional content in written words or in facial expressions of the same emotion. Disgust was chosen as the emotion of interest because it can be easily conveyed in words and faces and because it consistently activates a specific region in the brain, the anterior insula (for a review, see Gallese et al., 2004). Indeed, the anterior insula is activated by facial expressions of disgust (Phillips et al., 1997, 1998). Electrophysiological recordings with depth electrodes confirmed that the anterior, but not the posterior, insula was selectively activated by facial expressions of disgust (Krolak-Salmon et al., 2003). Lesions of the left insula resulted in a selective impairment of recognizing disgust in facial expressions, whereas the recognition of other emotions remained unaffected (Calder, Keane, Manes, Antoun, \& Young, 2000). The anterior insula not only does respond to facial expressions of disgust but also strongly responds to gustatory and olfactory stimuli (Small et al., 2003; Royet et al., 1999, 2000). Wicker et al. (2003) showed that precisely the same region of the anterior insula was selectively activated not only when people smelled disgusting odors but also when they looked at others smelling disgusting odors. Indeed, Iacoboni and Dapretto (2006) hypothesized that the anterior insula allows people to match their own emotions with that of others.

It should be noted, however, that the anterior insula is activated in a wide range of sensory and affective experiences, such as mothers viewing photos of their own child, seeing or making a smile, attending to pleasant music, and so on (for a review, see Craig, 2009). Recently, Corradi-Dell'Acqua, Tusche, Vuilleumier, and Singer (2016) compared the response of the insula to equally unpleasant experiences of disgust, pain, and unfairness. They found that the left anterior insula codes for more general properties of affective experiences common to pain, disgust, and unfairness, whereas the right anterior insula exhibited activity patterns specific for pain, disgust, and unfairness. The anterior insula has efferent projections to several structures that belong to the circuit of emotions, like to the amygdala, lateral OFC, olfactory cor- tex, ACC, and also STS. It receives input from OFC, olfactory cortex, ACC, and STS (Cerliani et al., 2012; Mesulam \& Mufson, 1982; Mufson \& Mesulam, 1982). Thus, although the predominant role of the anterior insula in processing disgust is undebatable, it should be acknowledged that disgust is likely to be processed in a network of affective systems that include OFC and mid-ACC.

In summary, in the first experiment, fMRI was used to find out whether reading disgust words in print would activate the same region as looking at disgusted faces (i.e., anterior insula). In the second experiment, repetitive TMS (rTMS) was applied over the anterior insula to look for a causal involvement of this region for the processing of disgust during reading.

\section{EXPERIMENT 1}

Two critical conditions were used in the present fMRI experiment. In the face condition, participants passively viewed short video clips of actors with either neutral or disgusted facial expressions (Figure 1). In the word condition, participants made lexical decisions on words (i.e., decide whether the presented letter string was a word or not), which were either disgusting or neutral. The embodied cognition view of word perception would predict that the processing of the disgust value of a written word includes the neural structure (outside the classic language network) that is in charge of processing disgust in other sensory modalities (e.g., odors, faces).

\section{Materials and Methods}

\section{Participants}

For the fMRI experiment, 40 participants were recruited, four of whom aborted the experiment and thus were not included in the analyses. The remaining 36 participants were right-handed German native speakers (23 women), had normal or corrected-to-normal vision, and ranged from 19 to 35 years old (mean $=24.5$ years old, $S D=$ 4.0 years old). They all provided informed written

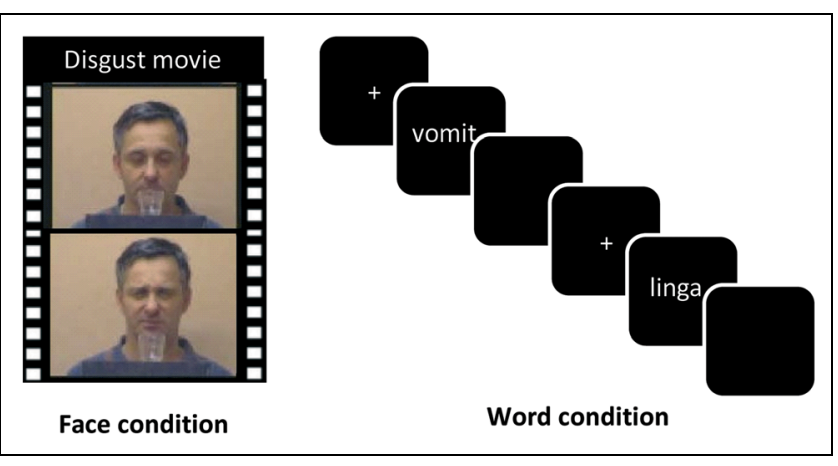

Figure 1. Processing emotional content in faces versus words. People viewed movie clips of actors who expressed either disgusted or neutral emotions (face condition), and they performed a lexical decision task on words with disgusting versus neutral content (word condition). 
consent before participation. The study was approved by the local ethics committee of Charité Berlin.

\section{Stimuli}

Face condition. To localize the anterior IC, participants were presented with 16 movie clips that showed actors smelling a transparent liquid in a glass. In half of the movies, the actors made a neutral face after having smelled the odor; in the other half, they made a disgusted facial expression. The movies were taken from Wicker et al. (2003).

Word condition. The stimulus set consisted of 50 disgust-related and 50 neutral German words taken from the Discrete Emotion Norms for Nouns-Berlin Affective Word List (Briesemeister, Kuchinke, \& Jacobs, 2011b) and 100 pronounceable nonwords created by changing one letter from real words that were not part of the stimulus set. Words and nonwords were matched with respect to stimulus length, measured in number of letters and number of syllables (all $t \mathrm{~s}<1$ ). The word material was selected from a pilot study in which disgust words produced the classic inhibitory disgust word effect (longer RTs and more errors) when compared with neutral words (Silva, Montant, Ponz, \& Ziegler, 2012; Briesemeister, Kuchinke, \& Jacobs, 2011a).

Disgust and neutral words were five to eight letters long and had a frequency below 30 occurrences per million according to the CELEX database (Baayen, Piepenbrock, \& van Rijn, 1993). Both word lists differed significantly with respect to disgust (mean disgust score $=3.1$ vs. $1.5, t>188$ ) and valence (mean valence score $=$ -1.6 vs. $-0.1, t>210$; taken from Vo et al. [2009]) but were controlled for arousal (3.4 vs. 3.2), imageability (4.5 vs. 4.6), number of letters (6.3 vs. 6.3), number of syllables (2.3 vs. 2.2), number of phonemes (5.4 vs. 5.6), frequency (3.9 vs. 3.3), orthographic neighborhood size (1.4 vs. 1.1), number of higher frequent orthographic neighbors (0.5 vs. 0.3), and mean bigram frequency (185.129 vs. 219.485 , all $t \mathrm{~s}<1$ ).

\section{fMRI Methods}

Functional and structural imaging was performed at the Dahlem Institute for Neuroimaging of Emotion with a Siemens Tim Trio 3-T using a 12-channel head coil (Siemens). The same settings were used for the face and word runs differing only in the number of acquired volumes (320 vs. 390, for faces and words, respectively ${ }^{1}$ ). Earplugs and headphones were used to attenuate the scanner noise, and form-fitting cushions were used to prevent the participants' head movements. High-resolution T1*-weighted anatomic reference images were acquired as a set of 176 continuous sagittal slices (repetition time $=$ $1900 \mathrm{msec}$, echo time $=2.52 \mathrm{msec}$, flip angle $=9^{\circ}$, matrix $=256 \times 256$, field of view $=256 \mathrm{~mm}, 1 \times 1 \times 1 \mathrm{~mm}$ voxels). Functional data were recorded in a single run with 390/320 volumes sensitive to BOLD contrast acquired with a T2-weighted gradient echo EPI sequence (repetition time $=2000 \mathrm{msec}$, echo time $=30 \mathrm{msec}$, flip angle $=70^{\circ}$, number of slices $=37$, slice thickness $=3 \mathrm{~mm}$, $64 \times 64$ matrix, field of view $=192 \mathrm{~mm}$ ). Five dummy scans were acquired at the beginning of each functional run before stimulus presentation. Low-frequency noise was removed with a high-pass filter $(128 \mathrm{sec})$.

The experimental sessions followed a strict order. Participants always performed the lexical decision task before the face processing task to avoid that, after having seen the face videos, they would draw too much attention to the specific emotional nature of the linguistic material (i.e., words that express disgust). In the lexical decision task, participants had to decide as fast and accurately as possible whether the presented letter string was a correct German word or not. Ten practice trials were run to familiarize the participants with the task. The experiment contained 200 trials. At the beginning of each trial, a fixation cross appeared in the center of the screen. After a jittered 2000- or 2500-msec presentation time, the fixation cross was replaced with the actual stimulus. Stimuli were presented for a maximum of 1500 msec in Arial font uppercase letters in white on a black background and disappeared after the participant pressed either the left (word response) or right (nonword response) button box, held in the dominant right hand. The lexical decision task was followed by the acquisition of the structural images. Finally, data for the face condition were acquired. Participants were instructed to simply watch the movies without any particular task. Movies were presented in a block design, following Wicker et al. (2003). In the first block, the eight neutral movie clips were shown, then followed by a block with the disgust movies, and so forth. Between blocks, a blank screen was presented for $1 \mathrm{sec}$. Movie duration was $3 \mathrm{sec}$. Movie presentation order was randomized within each block. Altogether, 12 blocks were shown per condition.

For preprocessing and statistical analysis, we used SPM8 software (SPM8; Wellcome Trust Center for Neuroimaging; Friston, Frith, Frackowiak, \& Turner, 1995), running in a MATLAB 7.6 environment (The MathWorks Inc.). Functional images were realigned, unwarped, corrected for geometric distortions using the fieldmap of each participant, and slice time corrected. The high-resolution structural T1-weighted image of each participant was processed and normalized with the VBM8 toolbox using default settings. Each structural image was segmented into gray matter, white matter, and CSF and denoised and warped into Montreal Neurological Institute (MNI) space by registering it to the DARTEL template provided by the VBM8 toolbox via the high-dimensional DARTEL (Ashburner, 2007) registration algorithm. On the basis of these steps, a skull stripped version of each image in native space was created. To normalize functional images into MNI space, the functional images were coregistered to the skull stripped structural image and the parameters 
from the DARTEL registration were used to warp the functional images, which were resampled to $3 \mathrm{~mm} \times$ $3 \mathrm{~mm} \times 3 \mathrm{~mm}$ voxels and smoothed with a 6-mm FWHM Gaussian kernel.

\section{Random Effects Statistical Data Analysis}

Preprocessed data were analyzed participant-by-participant using the standard general linear model approach of SPM8 with boxcar predictors. Two $t$ contrast maps were calculated: observation of disgust - neutral emotional expressions and reading of disgust - neutral words. Random effects analyses were applied to extrapolate statistical inferences into the general population. This two-stage analysis (second-order analysis) accounted first for intraparticipant (scan-to-scan) variance and second for betweenparticipant variance. At the group level, a voxel-by-voxel single-sample $t$ test was then performed to test if the contrast significantly differed from zero. Clusters were considered significant only if they were composed of at least 10 contiguous voxels, each of which have a $p<.05$ (FWE corrected).

\section{Conjunction Analyses}

Conjunction analyses between Contrasts A and B have been described as a method to test if both contrasts are different from zero in a particular voxel (Price \& Friston, 1997). Because of the implementation of the conjunction analysis in SPM8, the probability reported by such a conjunction analysis can pass a certain statistical threshold, despite the fact that one of the contrasts would not be significant if tested alone.

\section{Results}

\section{Behavioral Data}

Latencies for correct responses and accuracy rates were submitted to an ANOVA with Condition (disgust vs. neutral) as a within-participant factor. The results showed a highly significant inhibitory disgust effect on latencies (disgust: 810 msec; neutral: $762 \mathrm{msec} ; F(1,35)=93.679$, $p<.0001, \eta^{2}=.722$ ) and accuracy (disgust: 92.0\%; neutral: $\left.97.9 \% ; F(1,35)=54.045, p<.0001, \eta^{2}=.600\right)$.

\section{fMRI Data}

BOLD signal changes in the face and word conditions were analyzed using the same contrast (disgust - neutral). In the face condition, this contrast revealed significant BOLD signal changes in a network of brain areas including occipital, superior temporal, and parietal cortices; lateral pFC; anterior IC; and OFC for disgusting as compared with neutral faces (see Table 1). In the word condition, this contrast resulted in significant voxels in the left anterior insula/lateral $\mathrm{pFC}$ for disgusting as compared with neutral words (Table 2). The inverse contrast (neutral - disgust) yielded no significant clusters for neither words nor faces (at $p<.05$, FWE corrected for the whole-brain volume).

Most importantly, conjunction analyses revealed a significant cluster (127 voxels) of overlap between disgust effect for words and faces (see Figure 2). This cluster had three activation maxima (see Table 3 ). They were located in the left anterior insula and in the transition zone between the insula and the inferior frontal gyrus.

Table 1. Significant Differences in BOLD Signal for the Disgust-Neutral Contrast in the Face Condition

\begin{tabular}{lcccc}
\hline Brain Regions & MNI Coordinates & Peak Z & Cluster Size & $p_{\text {svc }}$ \\
\hline L Cerebellum & $-21,-82,-41$ & 5.99 & 129 & .000 \\
R Middle temporal gyrus & $54,-31,-8$ & 5.90 & 86 & .000 \\
R Supramarginal gyrus & $60,-43,28$ & 5.38 & 13 & .002 \\
R Precentral cortex & $45,5,46$ & 5.35 & 11 & .002 \\
R Middle temporal cortex & $51,2,-29$ & 5.29 & 11 & .003 \\
R Cerebellum & $18,-82,-38$ & 5.27 & 4 & .003 \\
R Inferior frontal gyrus, opercular & $48,14,28$ & 5.23 & 7 & .004 \\
L Supramarginal gyrus & $-48,-43,25$ & 5.17 & 4 & .006 \\
R Cerebellum crus 1 & $36,-64,-26$ & 5.15 & 6 & .006 \\
R Superior temporal pole & $54,11,-2$ & 5.14 & 12 & .007 \\
R Inferior frontal gyrus, opercular & $48,20,16$ & 5.14 & 3 & .007 \\
L Inferior frontal gyrus/anterior insula & $-45,11,-2$ & 5.12 & .008 \\
R Middle temporal gyrus & $51,-7,-17$ & 5.04 & .012 \\
\hline
\end{tabular}

Significant at $p<.05$, FWE corrected for the whole-brain volume. 
Table 2. Significant Differences in BOLD Signal for the Disgust-Neutral Contrast in the Word Condition

\begin{tabular}{lccr}
\hline Brain Regions & MNI Coordinates & Peak Z & Cluster Size \\
\hline L Inferior frontal gyrus/anterior insula & $-39,26,-11$ & 5.37 & 14 \\
L Inferior frontal gyrus, triangular & $-39,29,10$ & 5.18 & .002 \\
\hline
\end{tabular}

Significant at $p<.05$, FWE corrected for the whole-brain volume.

\section{Discussion}

The behavioral results replicated the classic inhibitory disgust word effect. That is, as in previous studies in French and German, disgust words produced longer RTs and more errors when compared with neutral words (Silva et al., 2012; Briesemeister et al., 2011a). In the fMRI study, conjunction analyses showed that the same region of the anterior insula was activated whether people looked at disgusted faces or read disgusting words $(x=-39, y=23, z=-14)$. Note that the almost exact location was obtained in Wicker et al.'s (2003) overlap analyses between observing facial expressions of disgust and actually smelling disgusting odorants $(x=-38, y=26, z=-6)$. Thus, compared with neutral words, disgust words activate a primary emotion brain area outside the classic language network that is involved in processing disgust in other sensory modalities. This result is in line with the predictions of the "hot" view of reading.

\section{EXPERIMENT 2}

The purpose of the second experiment was to find out whether the insula plays a causal role in processing dis- gust values during reading. This was done by investigating whether a stimulation of the anterior insula using rTMS would modulate the size of the disgust effect compared with the stimulation of a control site. The experiment was conducted with a new sample of participants. Before the rTMS study, they participated in an fMRI experiment, which served as a functional disgust localizer. This localizer was identical to the face condition of Experiment 1 described above. The localizer allowed us to identify for each participant the part of the left and right anterior insula that responded most strongly to disgust (Figure 4A). Before the lexical decision task, a 10-min rTMS train at $1 \mathrm{~Hz}$ (600 pulses) was applied over the individually identified regions of the left and right insula that responded most strongly to disgust in the localizer. A control stimulation was done using the vertex as a stimulation site. We predicted that rTMS should modulate the size of the disgust effect in the lexical decision task as compared with the control stimulation, but we had no specific hypothesis as to whether rTMS should reduce or increase the size of the inhibitory effect (see General Discussion).
Figure 2. Illustration of the overlap (conjunction) between the brain activation during the observation of facial expression of disgust and the reading of disgust words (compared with neutral faces and words).

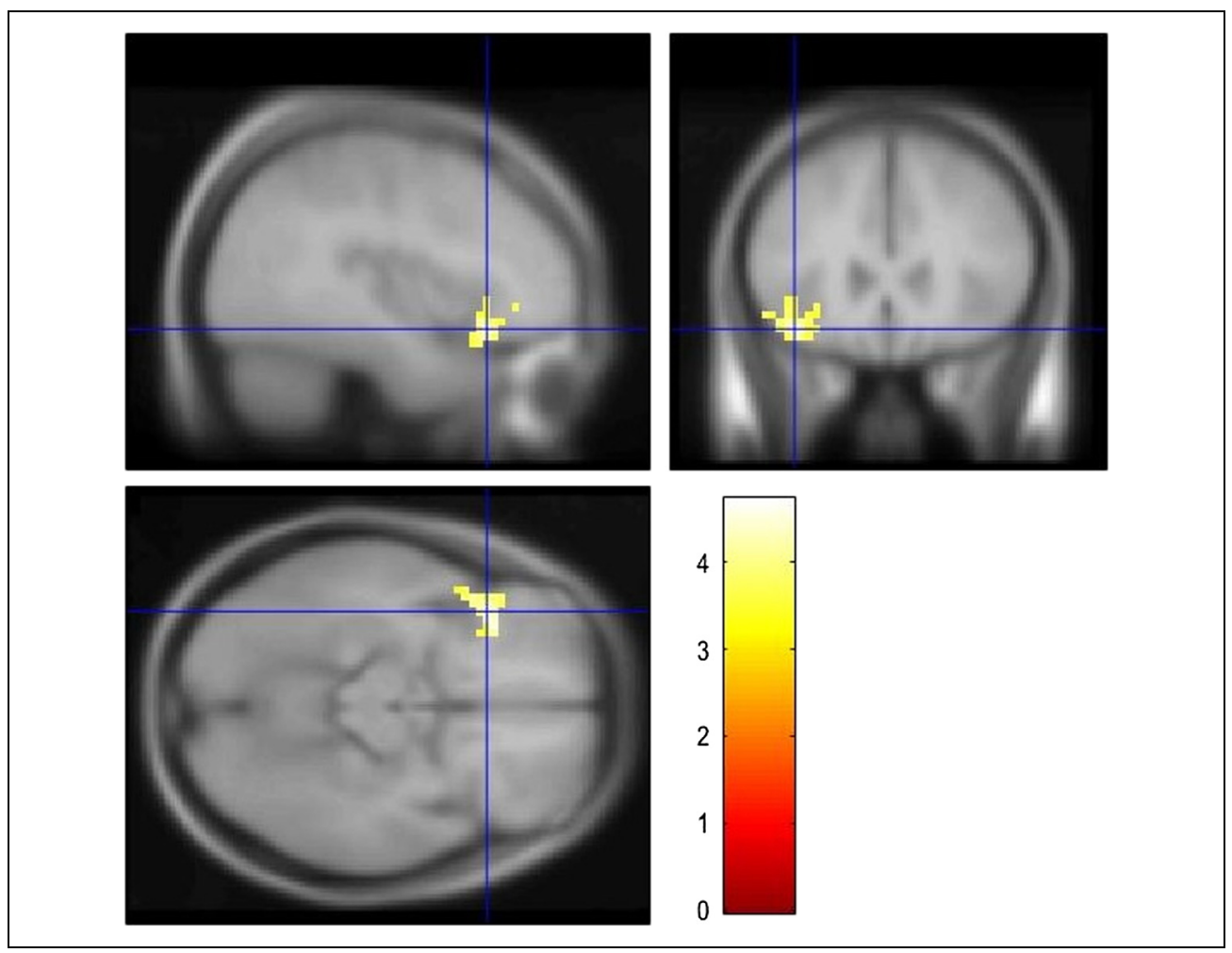


Table 3. Overlap in MNI Space between Observing Facial Expressions of Disgust and Reading Disgust Words

\begin{tabular}{|c|c|c|c|c|c|c|}
\hline \multirow[b]{2}{*}{ Brain Regions } & \multicolumn{3}{|c|}{ MNI Coordinates } & \multicolumn{3}{|c|}{ Disgust Effect ( $t$ Values) } \\
\hline & $x$ & $y$ & $z$ & Words & Faces & Conjunction \\
\hline L Anterior insula & -39 & 23 & -14 & 6.76 & 4.71 & 4.71 \\
\hline L Inferior frontal gyrus & -48 & 17 & -11 & 4.33 & 4.51 & 4.33 \\
\hline L Inferior frontal gyrus & -45 & 14 & -2 & 4.33 & 5.09 & 4.33 \\
\hline
\end{tabular}

Results show one significant cluster (127 voxels) in the left hemisphere with three peaks (i.e., activation maxima). The maximal $t$ score for each peak is presented separately for the word contrast, the face contrast, and the conjunction between the two.

\section{Methods}

\section{Participants}

An independent sample of 21 right-handed, native German participants was selected. Five participants had to be excluded from the analyses: Two participants showed no insula activation in the localizer, one responded randomly in the lexical decision task, and two participants withdrew in the course of the rTMS experiment. The remaining 16 participants (10 women) were between 19 and 28 years old (mean $=22.9$ years old, $S D=$ 2.7 years old). All participants provided written informed consent before participation. The experiment was approved by the local ethics committee of Charité Berlin.

\section{Procedure}

All participants participated in four separate sessions that were between 3 and 7 days apart. In the first session, we acquired structural MRI data and identified for each participant the part of the left and right anterior insula that responded most strongly to disgust (i.e., the disgust/ insula localizer). fMRI acquisition of the localizer was identical to the face condition described in Experiment 1. During that same session, EEG data from 14 scalp electrodes were recorded and checked by a physician to detect any potential risk of epileptic seizures.

During each of the remaining three sessions, a 10-min rTMS train at $1 \mathrm{~Hz}$ (600 pulses) was applied over one of the three stimulation sites: the left or right insula or the vertex (control site) just before the participants performed a lexical decision task. Individual fMRI localizers obtained during the first session were used in the TMS neuronavigation system to precisely position the coil for each participant The sequence of sessions (left, right, control) was randomized across participants in a singleblind crossover design. Participants performed the lexical decision task immediately after the stimulation in the same laboratory room. The task was identical to the one used in Experiment 1 (fMRI) except that the duration of the fixation cross was reduced to $200 \mathrm{msec}$ and the intertrial interval was fixed to $500 \mathrm{msec}$ (no jitter).
Figure 3. Coronal slice of the brain showing the right anterior insula (red arrow), the frontal operculum, and the inferior frontal gyrus (pars opercularis and pars triangularis). On the grid, 1 square $=1 \mathrm{~cm}$. The figure is taken from the Atlas of the Human Brain (http://www. thehumanbrain.info/).

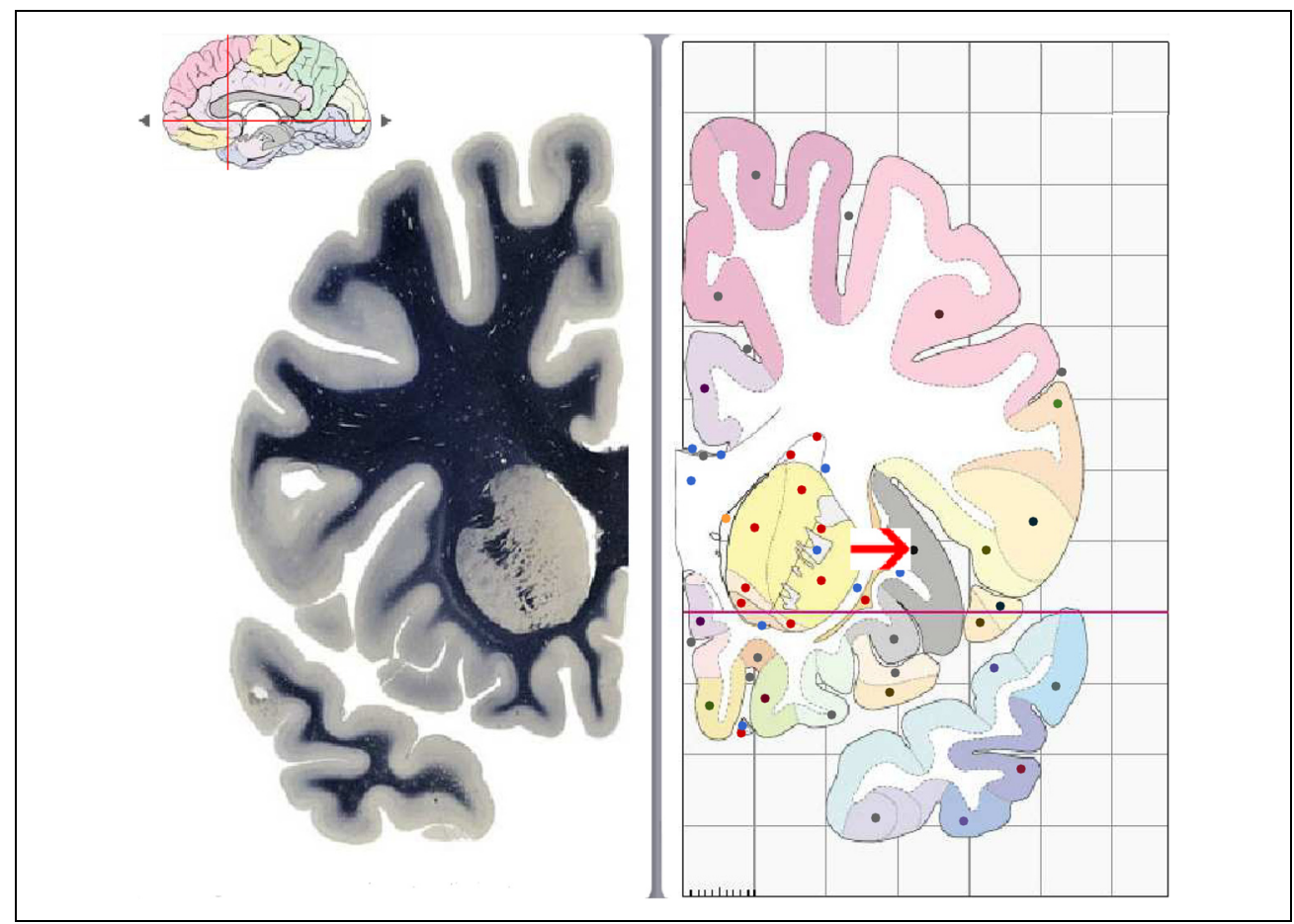


Figure 4. Main results of the rTMS study. (A) On the basis of the fMRI disgust localizer task, the anatomical region within the anterior IC that responded most strongly to the facial expression of disgust was selected for each individual participant (orange pointer) for rTMS. Depicted is an example showing the position of the coil over the left anterior IC. (B) Lexical decision latencies (milliseconds) on disgust words versus neutral words as a function of stimulation site (left insula, right insula, vertex). Error bars represent withinparticipant standard errors.
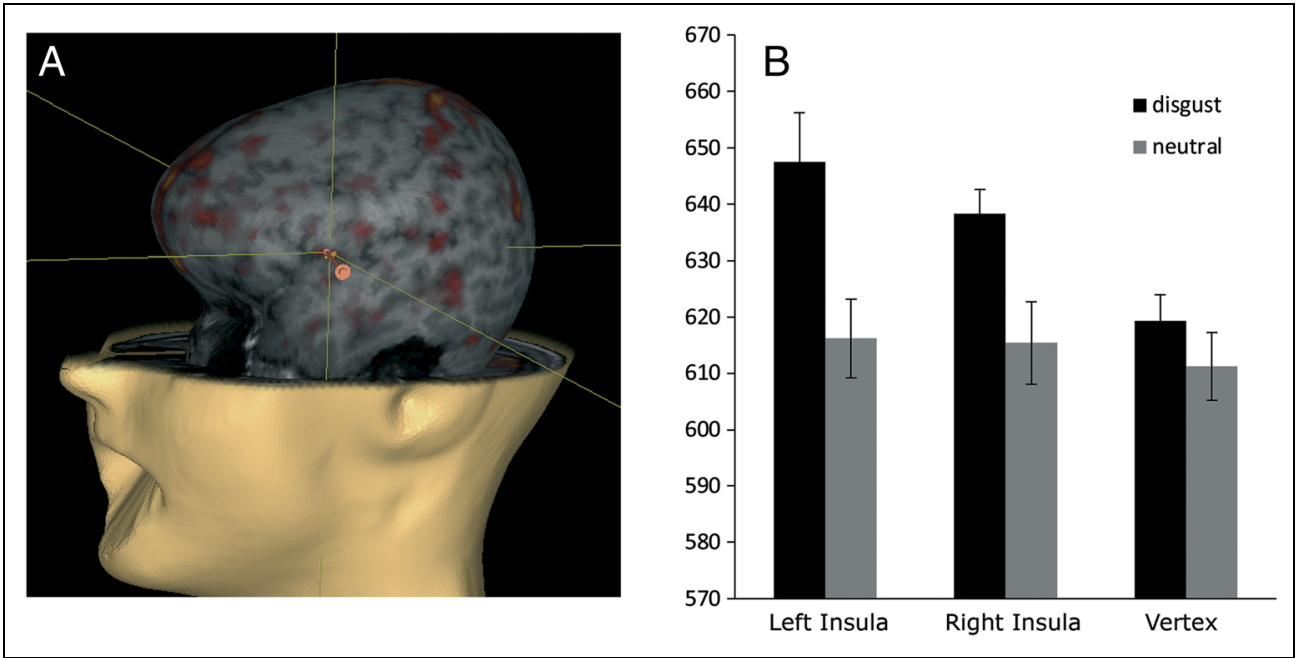

Biphasic magnetic stimulation was delivered with a Medtronic stimulator (MagPro X100 with MagOption; MagVenture) connected to a figure-eight coil (MCFB65). Neuronavigation (eXimia Navigated Brain Stimulation; Nexstim) was used to position the coil, based on the fMRI disgust localizer data, which were mapped on the participants' T1-weighted images. Surface electromyography was measured in the right first dorsal interosseus muscle of the hand to determine the resting motor threshold as the minimum intensity of stimulator output necessary to elicit motor potentials of at least $50-\mu \mathrm{V}$ peakto-peak amplitude in 5 of 10 stimulations. For this purpose, single-pulse TMS was applied over the primary motor cortex of the hand with intervals of at least $8 \mathrm{sec}$ to avoid carry-over effects. The resting motor threshold was measured for each participant at the beginning of the session. During the experiment, TMS intensity was set to $100 \%$ of that of the motor threshold, which corresponded on average to $48.87 \%$ of the maximum output stimulator intensity $(S D=6.72 \%)$. According to calculations from the neuronavigation system, magnetic stimulations at $49 \%$ of the maximum output stimulator induce a $65-\mathrm{V} / \mathrm{m}$ electric field in the primary motor cortex of the hand and $80 \mathrm{~V} / \mathrm{m}$ in the anterior insula. The magnetic field is stronger for the anterior insula than the motor cortex probably because the frontotemporal bone over the anterior insula is half as thick as the frontoparietal bone over the primary motor cortex (Mahinda \& Murty, 2009). Therefore, magnetic stimulation at $49 \%$ of the maximum output stimulator should be as efficient over the anterior insula as it is over the primary motor cortex of the hand. Although the insula is a rather deep structure that is at the limits of the TMS possibilities, previous studies have shown that deep structures, such as the ventral occipito-temporal cortex (average depth of $26.3 \mathrm{~mm}$ ), can be successfully reached with a standard figure-eight coil at 100\% motor threshold (Duncan, Pattamadilok, \& Devlin, 2010). As shown in Figure 3, the IC lies approximately $25 \mathrm{~mm}$ from the scalp including skull thickness
( $\sim 5 \mathrm{~mm}$ at the temporal lobe). Therefore, it is reasonable to assume that our stimulation parameters allowed us to reach the IC. The rTMS parameters were well within suggested safety guidelines (Wassermann, 1998). During stimulation, all participants wore earplugs.

\section{Results}

The data were analyzed in a $3 \times 2$ within-participant ANOVA with Site (left insula, right insula, vertex) and Condition (disgust vs. neutral) as within-participant factors. Error rates were fairly low (between 2\% and 6\%) and did not show any differences between conditions or interactions (all Fs $<1$ ). On RTs, however, the condition effect was highly significant, $F(1,15)=41.44, p<.0001$, partial $\eta^{2}=.734$, which reflects the fact that disgust words produced longer RTs than neutral words (i.e., the classic inhibitory disgust effect). Most importantly, there was a significant Site $\times$ Condition interaction, $F(2,30)=3.485$, $p<.049$, partial $\eta^{2}=.189$, reflecting the finding that the size of the disgust effect varied as a function of stimulation site: It was largest after stimulation of the left insula, intermediate after stimulation of the right insula, and smallest in the control condition (vertex). Indeed, $2 \times 2$ post hoc comparisons showed that the Site $\times$ Condition interaction was significant when the left insula was compared with the vertex, $F(1,15)=8.417, p<.011$, partial $\eta^{2}=.359$, but failed to reach significance when the right insula was compared with the vertex, $F(1,15)=3.138, p>.097$, partial $\eta^{2}=.173$. Yet, the direct comparison between effect sizes for the right and left insula failed to reach significance $(F<1)$. The main results are displayed in Figure 4B.

\section{Discussion}

The present rTMS experiment showed that repetitive stimulation of the left or right insula increased the size of the inhibitory disgust effect. Although the increase of the disgust effect seemed greater after stimulation of the 
left than the right insula, the difference was not statistically significant. However, compared with the control condition, the left insula stimulation produced a significant two-way interaction, whereas the two-way interaction failed to reach significance after right insula stimulation. This pattern was expected from the results of Experiment 1, which showed stronger effects of disgust in both the face and word conditions in the left anterior insula as compared with the right anterior insula. Indeed, most previous studies reported stronger activation of the left than the right insula in response to disgust (Small et al., 2003; Wicker et al., 2003; Royet et al., 1999, 2000; Sprengelmeyer, Rausch, Eysel, \& Przuntek, 1998), although at least one study has reported stronger activations to facial expressions of disgust in the right insula (Phillips et al., 1997). Recently, Corradi-Dell'Acqua et al. (2016) suggested that the right anterior insula might code for modality-specific representations of gustatory or nociceptive inputs, whereas the left insula might integrate the output of these modality-specific computations into more abstract representations of these events.

In contrast to Experiment 1, we did not obtain any disgust effect on error rates, neither a main effect of disgust nor a modulation of the disgust effect as a function of stimulation site. The absence of a main effect of disgust is probably due to the repetition of the same items across the three rTMS sessions, which was necessary to have a within-participant design. The explanation in terms of reduced effect sizes due to repetition is also supported by the fact that the disgust effect on RTs was smaller in Experiment 2 than in Experiment 1. The absence of an rTMS effect on error rate suggests that repetitive stimulation of either the left or right insula slows down processing times for words that "carry" emotional content but does not make people feel uncertain as to whether the stimulus was a word or not.

\section{GENERAL DISCUSSION}

The main findings can be summarized as follows. In the fMRI experiment, we found an overlap of the increased BOLD signal in the left anterior insula when people observe facial expressions of disgust and when they read words with disgusting content, as compared with emotionally neutral conditions. Because the anterior insula is specifically activated when people experience disgust or when they observe others experience disgust (Wicker et al., 2003), we can conclude that processing semantic content related to disgust during reading relies on parts of the same primary emotion network that is involved in the first- and third-person experiences of disgust (Gallese et al., 2004).

Furthermore, the rTMS results suggest that the left anterior insula is not an "innocent bystander" but is causally involved in processing emotional content related to disgust during reading. This finding is in line with results from intracranial recordings and surface EEG, which showed an activation of the left insula during the process- ing of disgust words as early as $200 \mathrm{msec}$ after the onset of the written word (Ponz et al., 2014), that is, during the initial stages of visual word recognition. These findings challenge the "cold" view of reading.

Before drawing strong theoretical conclusions, two major caveats need to be addressed. First, anterior insula lies deep in the lateral sulcus (Cerliani et al., 2012; Mesulam \& Mufson, 1982; Mufson \& Mesulam, 1982), which raises the question as to whether classic TMS can actually reach such a deep structure. As shown in the Methods section, we are fairly confident that the IC can be successfully reached with a standard figure-eight coil at 100\% motor threshold (Duncan et al., 2010). Moreover, estimations of the induced electric field suggest that the depth of the anterior insula is offset by the relatively reduced thickness of the tissues that cover that region. Most importantly, our results show that rTMS only affected the emotion words but not the neutral words (i.e., response latencies of neutral words were flat across conditions; see Figure $4 \mathrm{~B})$. Thus, we obviously managed to interfere with a network that is involved in emotional processing of words but not word recognition in general. Given that disgust has such a clear neural locus, the anterior insula (Jabbi, Bastiaansen, \& Keysers, 2008; Sprengelmeyer, 2007; Phillips et al., 1997), it is tempting to assume that our rTMS had indeed reached the insula. Note that the anterior insula receives input from the frontal operculum and the OFC (Cerliani et al., 2012; Mesulam \& Mufson, 1982; Mufson \& Mesulam, 1982) and our stimulation trajectory extended from the pars opercularis of the inferior frontal gyrus to the underneath frontal operculum terminating in the anterior part of the insula. Thus, it is also possible that our repetitive stimulation interfered with these adjacent structures that project into the anterior insula. If so, impoverished input to the anterior insula might have produced particular disruptive effects for disgust words. In any case, as argued above, an inadvertent stimulation of nearby language areas would not be sufficient to explain the present findings because we did not see any modulation of the response latencies for neutral words.

Second, if one "wipes out" the insula using rTMS, one might have expected to find smaller but not larger disgust effects. Indeed, if we believed that processing emotional content by the insula is some sort of an add-on process that interferes with "normal" word recognition, then we would expect response latencies to be reduced if we managed to inhibit the insula, thereby reducing the interference. However, if the neural representation of disgust words necessarily includes the coactivation of the insula as part of the neural assembly that codes the meaning of these words (e.g., Pulvermuller, 1999, 2005), then the inhibition of the insula would weaken the neural assembly. In that case, more time would be needed for the disgust word attractor to settle into a stable state, which would result in longer response latencies. Thus, the present finding of a larger disgust word effect when 
applying rTMS over the insula is in favor of the view that insula activation is a very part of the neural representation that codes the meaning of disgust words.

The present results are broadly compatible with theories of embodied emotion (Niedenthal, 2007), mirror neurons (Gallese et al., 2004), and neural reuse (Anderson, 2010), which assume that phylogenetically ancient brain structures that process basic emotions in all mammals (e.g., the insula) actively participate in high-level cognitive skills, such as language, that are developed extensively in humans. It is worth noting that neural reuse is a general principle that applies not only to the language of emotions but also to language itself. For example, the understanding of action verbs, such as "kick," "pick," and "lick," relies on activity in the corresponding action systems (Pulvermuller, 2005); the understanding of taste words, like "salt," implies activity in gustatory cortex (Barrós-Loscertales et al., 2012); and the understanding of odor words, such as "cinnamon," activates the primary olfactory cortex (González et al., 2006). Indeed, language appears more widely scattered in the brain than other cognitive functions, as shown in a meta-analysis of 472 imaging studies (Anderson, 2008), which suggests that, in the course of evolution, language development itself might have relied on the reuse of a variety of ancient brain structures. Similar principles apply to reading development during childhood. The reading network is not created from scratch, but its development relies heavily on the reuse of the spoken language network (Goswami, 2015; Ziegler, Perry, \& Zorzi, 2014; Goswami \& Ziegler, 2006), as seen in literally all brain imaging studies on reading (Cavalli et al., 2016; Martin, Schurz, Kronbichler, \& Richlan, 2015). Thus, at the phylogenetical and developmental level, neural reuse seems to be a powerful explanatory mechanism for building higher-level cognitive functions.

The idea that both spoken and written words are embodied stimuli with the potential to elicit overt and covert sensory-motor and affective responses goes back to the early theoreticians of language, such as Freud (1891) or Bühler (1934). For example, Bühler introduced the notion of Sphärengeruch (spheric fragrance of words), according to which words have a substance and the actions they serve-speaking, reading, thinking, and feeling - are themselves substance controlled. The word Radieschen (garden radish) can evoke red or white color impressions, crackling sounds, or earthy smells and spicy tastes. The renaissance of Bühler's ideas in recent theories of symbol grounding, embodied cognition, or neural reuse can explain why evolutionary recent cultural objects, such as written words, can evoke basic emotions as well as aesthetic feelings and activate affective processing networks at the neuronal level.

\section{Acknowledgments}

This research was supported by a French-German ANR/DFG grant (ANR-10-FRAL-005-01) awarded to J. C. Z. and A. M. J.
Parts of this study were carried out within the Labex BLRI (ANR-11-LABX-0036) and the Convergence Institute ILCB (ANR-16-CONV-0002) and, as such, have benefited from support from the French government, managed by the French National Agency for Research (ANR) and the Excellence Initiative of Aix-Marseille University (A*MIDEX).

Reprint requests should be sent to Johannes C. Ziegler, Laboratoire de Psychologie Cognitive, Aix Marseille Université, 3, Place Victor Hugo, Bat 9, Case D, 13331 Marseille Cedex 3, France, or via e-mail: Johannes.ziegler@univ-amu.fr.

\section{Note}

1. The volumes were slightly different because we aimed for the shortest in-scanner time possible. Other than that, there was no particular rationale for this choice.

\section{REFERENCES}

Anderson, M. L. (2008). Circuit sharing and the implementation of intelligent systems. Connection Science, 20, 239-251.

Anderson, M. L. (2010). Neural reuse: A fundamental organizational principle of the brain. Behavioral and Brain Sciences, 33, 245-266.

Ashburner, J. (2007). A fast diffeomorphic image registration algorithm. Neuroimage, 38, 95-113.

Baayen, R. H., Piepenbrock, R., \& van Rijn, H. (1993). The CELEX lexical database (CD-ROM). Philadelphia, PA: Linguistic Data Consortium, University of Pennsylvania.

Barrós-Loscertales, A., González, J., Pulvermüller, F., VenturaCampos, N., Bustamante, J. C., Costumero, V., et al. (2012). Reading salt activates gustatory brain regions: fMRI evidence for semantic grounding in a novel sensory modality. Cerebral Cortex, 22, 2554-2563.

Barsalou, L. W. (2008). Grounded cognition. Annual Review of Psychology, 59, 617-645.

Briesemeister, B. B., Kuchinke, L., \& Jacobs, A. M. (2011a). Discrete emotion effects on lexical decision response times. PLoS One, 6, e23743.

Briesemeister, B. B., Kuchinke, L., \& Jacobs, A. M. (2011b). Discrete emotion norms for nouns: Berlin Affective Word List (DENN-BAWL). Behavior Research Methods, 43, 441-448.

Bühler, K. (1934). Sprachtheorie. Stuttgart, Germany: Lucius und Lucius.

Calder, A. J., Keane, J., Manes, F., Antoun, N., \& Young, A. W. (2000). Impaired recognition and experience of disgust following brain injury. Nature Neuroscience, 3, 1077-1078.

Cavalli, E., Cole, P., Badier, J. M., Zielinski, C., Chanoine, V., \& Ziegler, J. C. (2016). Spatiotemporal dynamics of morphological processing in visual word recognition. Journal of Cognitive Neuroscience, 28, 1228-1242.

Cerliani, L., Thomas, R. M., Jbabdi, S., Siero, J. C., Nanetti, L., Crippa, A., et al. (2012). Probabilistic tractography recovers a rostrocaudal trajectory of connectivity variability in the human insular cortex. Human Brain Mapping, 33, 2005-2034.

Corradi-Dell'Acqua, C., Tusche, A., Vuilleumier, P., \& Singer, T. (2016). Cross-modal representations of first-hand and vicarious pain, disgust and fairness in insular and cingulate cortex. Nature Communications, 7, 10904.

Craig, A. D. (2009). How do you feel-Now? The anterior insula and human awareness. Nature Review Neuroscience, 10, 59-70.

Dehaene, S., \& Cohen, L. (2011). The unique role of the visual word form area in reading. Trends in Cognitive Sciences, 15, 254-262. 
Duncan, K. J., Pattamadilok, C., \& Devlin, J. T. (2010). Investigating occipito-temporal contributions to reading with TMS. Journal of Cognitive Neuroscience, 22, 739-750.

Freud, S. (1891). Zur Auffassung der Aphasien: Eine kritische Studie [On aphasia: A critical study]. Wien, Austria: Deuticke.

Friston, K. J., Frith, C. D., Frackowiak, R. S., \& Turner, R. (1995). Characterizing dynamic brain responses with fMRI: A multivariate approach. Neuroimage, 2, 166-172.

Gallese, V., Keysers, C., \& Rizzolatti, G. (2004). A unifying view of the basis of social cognition. Trends in Cognitive Sciences, 8, 396-403.

González, J., Barros-Loscertales, A., Pulvermüller, F., Meseguer, V., Sanjuán, A., Belloch, V., et al. (2006). Reading cinnamon activates olfactory brain regions. Neuroimage, 32, 906-912.

Goswami, U. (2015). Sensory theories of developmental dyslexia: Three challenges for research. Nature Review Neuroscience, 16, 43-54.

Goswami, U., \& Ziegler, J. C. (2006). A developmental perspective on the neural code for written words. Trends in Cognitive Sciences, 10, 142-143.

Iacoboni, M., \& Dapretto, M. (2006). The mirror neuron system and the consequences of its dysfunction. Nature Review Neuroscience, 7, 942-951.

Jabbi, M., Bastiaansen, J., \& Keysers, C. (2008). A common anterior insula representation of disgust observation, experience and imagination shows divergent functional connectivity pathways. PLoS One, 3, e2939.

Jacobs, A. M., Vo, M. L., Briesemeister, B. B., Conrad, M., Hofmann, M. J., Kuchinke, L., et al. (2015). 10 years of BAWLing into affective and aesthetic processes in reading: What are the echoes? Frontiers in Psychology, 6, 714.

Jacobs, A. M., \& Ziegler, J. C. (2015). Visual word recognition, neurocognitive psychology of. In J. D. Wright (Ed.), International encyclopedia of the social \& behavioral sciences (2nd ed., pp. 214-219). Oxford: Elsevier.

Krolak-Salmon, P., Henaff, M. A., Isnard, J., Tallon-Baudry, C., Guenot, M., Vighetto, A., et al. (2003). An attention modulated response to disgust in human ventral anterior insula. Annals of Neurology, 53, 446-453.

LaBar, K. S., Gatenby, J. C., Gore, J. C., LeDoux, J. E., \& Phelps, E. A. (1998). Human amygdala activation during conditioned fear acquisition and extinction: A mixed-trial fMRI study. Neuron, 20, 937-945.

Mahinda, H. A. M., \& Murty, O. P. (2009). Variability in thickness of human skull bones and sternum-An autopsy experience. Journal of Forensic Medicine and Toxicology, 26, 26-31.

Mahon, B. Z., \& Caramazza, A. (2008). A critical look at the embodied cognition hypothesis and a new proposal for grounding conceptual content. Journal of Physiology, Paris, 102, 59-70.

Martin, A., Schurz, M., Kronbichler, M., \& Richlan, F. (2015) Reading in the brain of children and adults: A meta-analysis of 40 functional magnetic resonance imaging studies. Human Brain Mapping, 36, 1963-1981.

McRae, K., de Sa, V. R., \& Seidenberg, M. S. (1997). On the nature and scope of featural representations of word meaning. Journal of Experimental Psychology: General, 126, 99-130.

Mesulam, M. M., \& Mufson, E. J. (1982). Insula of the old world monkey: I. Architectonics in the insulo-orbito-temporal component of the paralimbic brain. Journal of Comparative Neurology, 212, 1-22.

Mufson, E. J., \& Mesulam, M. M. (1982). Insula of the old world monkey: II. Afferent cortical input and comments on the claustrum. Journal of Comparative Neurology, 212, 23-37.
Niedenthal, P. M. (2007). Embodying emotion. Science, 316, 1002-1005.

Pexman, P. M., Holyk, G. G., \& Monfils, M. H. (2003). Numberof-features effects and semantic processing. Memory $\varepsilon$ Cognition, 31, 842-855.

Phillips, M. L., Young, A. W., Scott, S. K., Calder, A. J., Andrew, C., Giampietro, V., et al. (1998). Neural responses to facial and vocal expressions of fear and disgust. Proceedings. Biological Sciences, 265, 1809-1817.

Phillips, M. L., Young, A. W., Senior, C., Brammer, M., Andrew, C., Calder, A. J., et al. (1997). A specific neural substrate for perceiving facial expressions of disgust. Nature, 389, 495-498.

Ponz, A., Montant, M., Liegeois-Chauvel, C., Silva, C., Braun, M., Jacobs, A. M., et al. (2014). Emotion processing in words: A test of the neural re-use hypothesis using surface and intracranial EEG. Social Cognitive and Affective Neuroscience, 9. 619-627.

Price, C. J. (2012). A review and synthesis of the first 20 years of PET and fMRI studies of heard speech, spoken language and reading. Neuroimage, 62, 816-847.

Price, C. J., \& Friston, K. J. (1997). Cognitive conjunction: A new approach to brain activation experiments. Neuroimage, 5 , 261-270.

Pulvermuller, F. (1999). Words in the brain's language. The Behavioral and Brain Sciences, 22, 253-279.

Pulvermuller, F. (2005). Brain mechanisms linking language and action. Nature Review Neuroscience, 6, 576-582.

Royet, J. P., Koenig, O., Gregoire, M. C., Cinotti, L., Lavenne, F., Le Bars, D., et al. (1999). Functional anatomy of perceptual and semantic processing for odors. Journal of Cognitive Neuroscience, 11, 94-109.

Royet, J. P., Zald, D., Versace, R., Costes, N., Lavenne, F., Koenig, O., et al. (2000). Emotional responses to pleasant and unpleasant olfactory, visual, and auditory stimuli: A positron emission tomography study. Journal of Neuroscience, 20, 7752-7759.

Silva, C., Montant, M., Ponz, A., \& Ziegler, J. C. (2012). Emotions in reading: Disgust, empathy and the contextual learning hypothesis. Cognition, 125, 333-338.

Small, D. M., Gregory, M. D., Mak, Y. E., Gitelman, D., Mesulam, M. M., \& Parrish, T. (2003). Dissociation of neural representation of intensity and affective valuation in human gustation. Neuron, 39, 701-711.

Sprengelmeyer, R. (2007). The neurology of disgust. Brain, 130, 1715-1717.

Sprengelmeyer, R., Rausch, M., Eysel, U. T., \& Przuntek, H. (1998). Neural structures associated with recognition of facial expressions of basic emotions. Proceedings. Biological Sciences, 265, 1927-1931.

Vo, M. L., Conrad, M., Kuchinke, L., Urton, K., Hofmann, M. J., \& Jacobs, A. M. (2009). The Berlin Affective Word List Reloaded (BAWL-R). Behavior Research Methods, 41, 534-538.

Wassermann, E. M. (1998). Risk and safety of repetitive transcranial magnetic stimulation: Report and suggested guidelines from the International Workshop on the Safety of Repetitive Transcranial Magnetic Stimulation, June 5-7, 1996. Electroencephalography and Clinical Neurophysiology, 108, 1-16.

Wicker, B., Keysers, C., Plailly, J., Royet, J. P., Gallese, V., \& Rizzolatti, G. (2003). Both of us disgusted in My insula: The common neural basis of seeing and feeling disgust. Neuron, 40, 655-664.

Ziegler, J. C., Perry, C., \& Zorzi, M. (2014). Modelling reading development through phonological decoding and selfteaching: Implications for dyslexia. Philosophical Transactions of the Royal Society of London, Series B, Biological Sciences, 369, 20120397. 